\title{
A combined approach for object detection and deconvolution
}

\author{
J.-L. Starck ${ }^{1,2}$, A. Bijaoui ${ }^{3}$, I. Valtchanov ${ }^{2}$, and F. Murtagh ${ }^{4,5}$ \\ 1 Statistics Department, Stanford University, Sequoia Hall, Stanford, CA 94305, U.S.A. \\ 2 SEI-SAP/DAPNIA, CEA-Saclay, 91191 Gif-sur-Yvette Cedex, France \\ 3 Observatoire de la Côte d'Azur, BP. 229, 06394 Nice Cedex 4, France \\ 4 School of Computer Science, Queen's University of Belfast, Belfast BT7 1NN, Northern Ireland \\ 5 Observatoire Astronomique, 11 rue de l'Université, 67000 Strasbourg, France
}

Received July 7; accepted August 29, 2000

\begin{abstract}
The Multiscale Vision Model is a recent object detection method, based on the wavelet transform. It allows us to extract all objects contained in an image, whatever their size or their shape. From each extracted object, information concerning flux or shape can easily be determined. We show that such an approach can be combined with deconvolution, leading to the reconstruction of deconvolved objects. We discuss the advantages of this approach, such as how we can perform deconvolution with a space-variant point spread function. We present a range of examples and applications, in the framework of the ISO, XMM and other projects, to illustrate the effectiveness of this approach.
\end{abstract}

Key words: methods: data analysis — techniques: image processing

\section{Introduction}

Astronomical images contain typically a large set of pointlike sources (the stars), some quasi point-like objects (faint galaxies, double stars) and some complex and diffuse structures (galaxies, nebulae, planetary stars, clusters, etc.). These objects are often hierarchically organized: star in a small nebula, itself embedded in a galaxy arm, itself included in a galaxy, and so on.

A standard approach to source detection consists of the following four steps:

- Background estimation;

- Noise estimation;

- Detection where flux $>$ Background $+k^{*}$ Noise;

- Gaussian or PSF (point source function) fitting to derive the photometry.

Send offprint requests to: J.-L. Starck, e-mail: jstarck@cea.fr

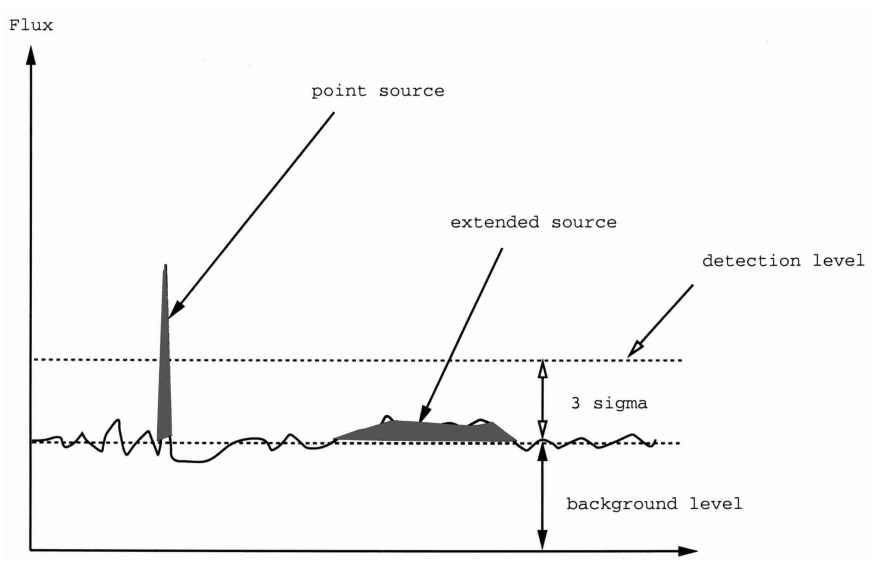

Fig. 1. Example of astronomical data: a point source and an extended source are shown, with noise and background. The extended object, which can be detected by eye, is undetected by a standard detection approach

Faint extended objects may be lost by this standard approach. Figure 1 shows a typical example where a faint extended object is under the detection limit. In order to detect faint objects, whatever their sizes, Bijaoui (1993) proposed the Multiscale Vision Model (MVM).

A vision model is defined as the sequence of operations required for automated image analysis. Taking into account the scientific purposes, the characteristics of the objects and the existence of hierarchical structures, astronomical images need specific vision models. This is also the case in many other fields, such as remote sensing, hydrodynamic flows, or biological studies. Specific vision models have been implemented for these kinds of images.

We first introduce the MVM as defined in Bijaoui \& Rué (1995). Then we describe how deconvolution can be combined with object reconstruction, and show that such an approach improves the photometry of the reconstructed 
objects, helps with object identification, and can be very helpful for deconvolution with a space-variant PSF.

\section{Multiscale vision model}

\subsection{Introduction}

The wavelet transform of an image by the à trous algorithm produces, at each scale $j$, a set $\left\{w_{j}\right\}$. This has the same number of pixels as the image. The original image $c_{0}$ can be expressed as the sum of all the wavelet scales and the smoothed array $c_{\mathrm{p}}$ by the expression $c_{0}=c_{\mathrm{p}}+$ $\sum_{j=1}^{\mathrm{p}} w_{j}$. A pixel at position $x, y$ can be expressed also as the sum of all the wavelet coefficients at this position, plus the smoothed array: $c_{0}(x, y)=c_{\mathrm{p}}(x, y)+\sum_{j=1}^{\mathrm{p}} w_{j}(x, y)$.

After applying the wavelet transform on the image, we have to detect, to extract, to measure and to recognize the significant structures. This is done by first computing the multiresolution support of the image, and by applying a segmentation scale by scale. The wavelet space of a $2 \mathrm{D}$ direct space is a $3 \mathrm{D}$ one. An object has to be defined in this space. A general idea for object definition lies in the connectivity property. An object occupies a physical region, and in this region we can join any pixel to other ones. Connectivity in direct space has to be transported to wavelet transform space (WTS). In order to define the objects we have to identify the WTS pixels we can attribute to the objects. We describe in this section the different steps of this method.

\subsection{Definition}

The Multiscale Vision Model (MVM) (Bijaoui \& Rué 1995; Rué \& Bijaoui 1997) described an object as a hierarchical set of structures. It uses the following definitions:

- significant wavelet coefficient: a wavelet coefficient is significant when its absolute value is above a given detection limit. The detection limit depends on the noise model (Gaussian noise, Poisson noise, and so on). See Starck et al. (1998) for a full description of the noise modelling;

- structure: a structure $\mathcal{S}_{j}$ is a set of significant connected wavelet coefficients at the same scale $j$;

- object: an object is a set of structures;

- object scale: the scale of an object is given by the scale of the maximum of its wavelet coefficients;

- interscale relation: the criterion allowing us to connect two structures into a single object is called the "interscale relation";

- sub-object: a sub-object is a part of an object. It appears when an object has a local wavelet maximum. Hence, an object can be composed of several subobjects. Each sub-object can also be analysed.

\subsection{The multiresolution support and its segmentation}

A multiresolution support of an image describes in a logical or Boolean way if an image $I$ contains information at a given scale $j$ and at a given position $(x, y)$. If $M^{(I)}(j, x, y)=1$ (or $=$ true), then $I$ contains information at scale $j$ and at the position $(x, y) . M$ depends on several parameters:

- The input image;

- The algorithm used for the multiresolution decomposition;

- The noise;

- All additional constraints we want the support to satisfy.

Such a support results from the data, the treatment (noise estimation, etc.), and from knowledge on our part of the objects contained in the data (size of objects, linearity, etc.). In the most general case, a priori information is not available to us.

The multiresolution support of an image is computed in several steps:

- Step one is to compute the wavelet transform of the image;

- Binarization of each scale leads to the multiresolution support (the binarization of an image consists of assigning to each pixel a value only equal to 0 or 1 );

- A priori knowledge can be introduced by modifying the support.

This last step depends on the knowledge we have of our images. For instance, if we know there is no interesting object smaller or larger than a given size in our image, we can suppress, in the support, anything which is due to that kind of object. This can often be done conveniently by the use of mathematical morphology. In the most general setting, we naturally have no information to add to the multiresolution support.

The multiresolution support will be obtained by detecting at each scale the significant coefficients. The multiresolution support is defined by:

$M(j, x, y)= \begin{cases}1 & \text { if } w_{j}(x, y) \text { is significant } \\ 0 & \text { if } w_{j}(x, y) \text { is not significant. }\end{cases}$

In the case of Gaussian noise, it suffices to compare the wavelet coefficients $w_{j}(x, y)$ to a threshold level $t_{j} . t_{j}$ is generally taken equal to $k \sigma_{j}$, where $\sigma_{j}$ is the noise standard deviation at scale $j$, and $k$ is chosen between 3 and 5 . The value of 3 corresponds to a probability of false detection of 0.0027 for Gaussian statistics. If $w_{j}(x, y)$ is small, then it is not significant and could be due to noise. If $w_{j}(x, y)$ is large, it is significant:

if $\left|w_{j}\right| \geq t_{j}$ then $w_{j}$ is significant if $\left|w_{j}\right|<t_{j}$ then $w_{j}$ is not significant.

Many other kinds of noise can be considered in the wavelet space. See (Starck et al. 1998) for a review. 

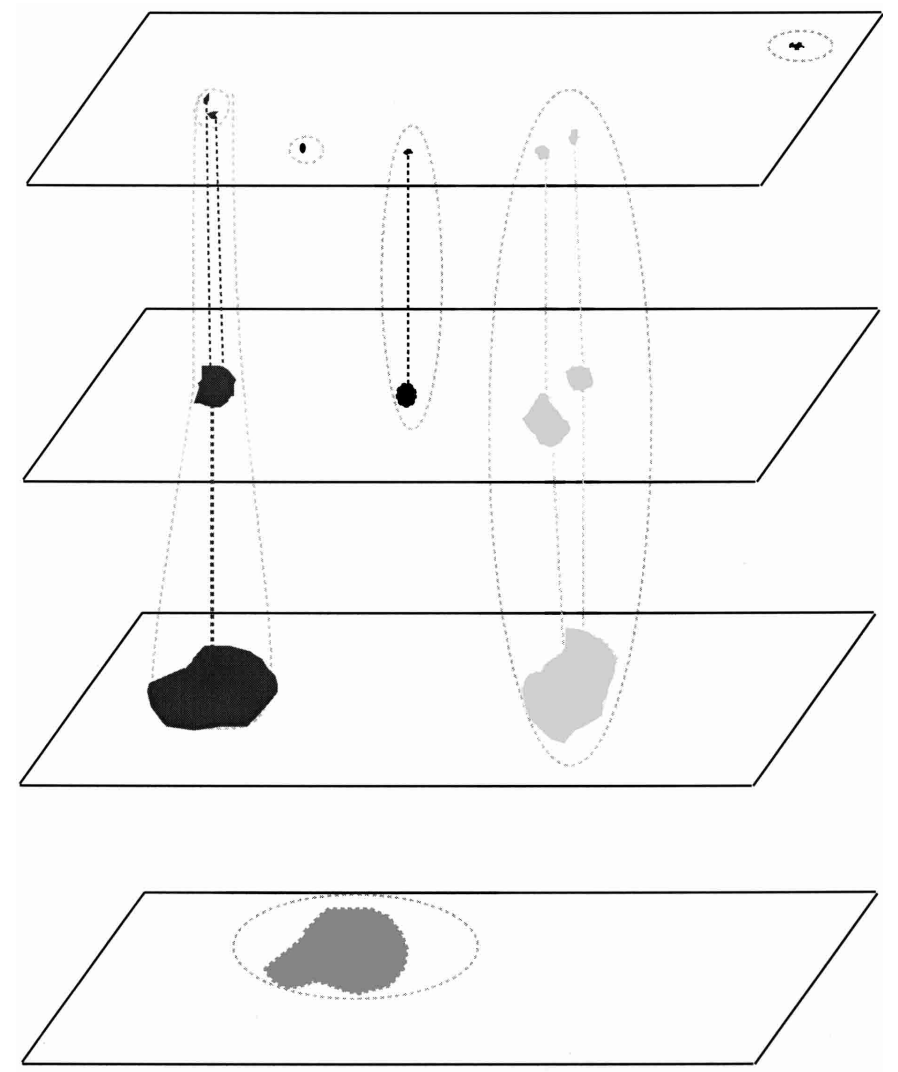

Fig. 2. Example of connectivity in wavelet space: contiguous significant wavelet coefficients form a structure, and following an interscale relation, a set of structures form an object. Two structures $S_{j}, S_{j+1}$ at two successive scales belong to the same object if the position pixel of the maximum wavelet coefficient value of $S_{j}$ is included in $S_{j+1}$

\section{Multiresolution support segmentation}

The segmentation consists of labelling a boolean image (0 or 1). Each group of connected pixels having a "1" value gets a label value between 1 and $L_{\max }, L_{\max }$ being the number of groups. This process is repeated at each scale of the multiresolution support. We define a "structure" $S_{j}^{i}$ as the group of connected significant pixels which has the label $i$ at a given scale $j$.

\subsection{Interscale connectivity graph}

An object is described as a hierarchical set of structures. The rule which allows us to connect two structures into a single object is called "interscale relation". Figure 2 shows how several structures at different scales are linked together, and form objects. We have now to define the interscale relation: let us consider two structures at two successive scales, $S_{j}^{k}$ and $S_{j+1}^{l}$. Each structure is located in one of the individual images of the decomposition and corresponds to a region in this image where the signal is significant. Denoting $p_{\mathrm{m}}$ the pixel position of the maximum wavelet coefficient value of $S_{j}^{k}, S_{j}^{k}$ is said to be connected to $S_{j+1}^{l}$ if $S_{j+1}^{l}$ contains the pixel position $p_{\mathrm{m}}$ (i.e. the maximum position of the structure $S_{j}^{k}$ must also be contained in the structure $\left.S_{j+1}^{l}\right)$. Several structures appearing in successive wavelet coefficient images can be connected in such a way, which we call an object in the interscale connectivity graph.

\subsection{Reconstruction}

Hence, a set of structures defines an object $W=\left\{S_{j}^{k}, \ldots S_{j^{\prime}}^{k^{\prime}}\right\}$ which can be reconstructed separately from other objects. The coaddition of all reconstructed objects is a filtered version of the input data.

The reconstruction problem consists of searching for a signal $O$ such that its wavelet coefficients are the same as those of the detected structures. If $\mathcal{T}$ describes the wavelet transform operator, and $P_{\mathrm{w}}$ the projection operator in the subspace of the detected coefficients (i.e. having set to zero all coefficients at scales and positions where nothing was detected), the solution is found by minimization of

$J(O)=\left\|W-\left(P_{\mathrm{w}} \circ \mathcal{T}\right) O\right\|$

where $W$ represents the detected wavelet coefficients of the data. More details can be found in Bijaoui \& Rué (1995).

\section{Detection and deconvolution}

\subsection{Introduction}

The PSF is not needed with MVM. This is an advantage when the PSF is unknown, or difficult to estimate, which happens relatively often when it is space-variant. However, when the PSF is well-determined, it becomes a drawback because known information is not used for the object reconstruction. This can lead to systematic errors in the photometry, which depends on the PSF and on the source signal to noise ratio. In order to preempt such a bias, a kind of calibration must be performed using simulations (Starck et al. 1999). This section shows how the PSF can be used in the MVM, leading to a deconvolution.

\subsection{The deconvolution problem}

Consider an image characterized by its intensity distribution (the "data") $I(x, y)$, corresponding to the observation of a "real image" $O(x, y)$ through an optical system. If the imaging system is linear and shift-invariant, the relation between the object and the image in the same coordinate frame is a convolution:

$I(x, y)=(O * P)(x, y)+N(x, y)$

$P(x, y)$ is the PSF of the imaging system, and $N(x, y)$ is additive noise. In practice $O * P$ is subject to nonstationary noise. We want to determine $O(x, y)$ knowing 
$I(x, y)$ and $P(x, y)$. This inverse problem has led to a large amount of work, the main difficulties being the existence of: (i) a cut-off frequency of the PSF, and (ii) the noise.

Equations (3) is always an ill-posed problem. This means that there is no unique least-squares solution of minimal norm $\|I(x, y)-P(x, y) * O(x, y)\|$, and some constraints must be added in order to regularize the problem (Gonzalez 1993; Pratt 1991; Starck 1998). Once the deconvolved image is obtained, it is generally difficult to know what the noise level is in the deconvolved image, and hence it becomes impossible to detect the objects with a confidence interval. For this reason, astronomers generally prefer to apply the detection on non-deconvolved images. Another argument against deconvolution is that is has been shown that some regularization methods like MEM (Narayan \& Nityananda 1986) affect the photometry.

\subsection{Object reconstruction using the PSF}

A reconstructed and deconvolved object can be obtained by searching for a signal $O$ such that the wavelet coefficients of $P * O$ are the same as those of the detected structures. If $\mathcal{T}$ describes the wavelet transform operator, and $P_{\mathrm{w}}$ the projection operator in the subspace of the detected coefficients, the solution is found by minimization of

$J(O)=\left\|W-\left(P_{\mathrm{w}} \circ \mathcal{T}\right) P * O\right\|$

where $W$ represents the detected wavelet coefficients of the data, and $P$ is the PSF. In this approach, each object is deconvolved separately. The flux related to the extent of the PSF will be taken into account. For point sources, the solution will be close to that obtained by PSF fitting. This problem is also different from global deconvolution in the sense that it is well constrained. Except for the positivity of the solution which is always true and must be used, no other constraint is needed. This is due to the fact that the reconstruction is performed from a small set of wavelet coefficients (those above a detection limit). The number of objects are the same as those obtained by the MVM, but the photometry and the morphology is different. The astrometry may also be affected.

\subsection{The algorithm}

Any minimizing method can be used to obtain the solution $O$. Since we did not find any problem of convergence, noise amplification, or ringing effect, we chose the van Cittert method on the grounds of its simplicity. For each detected object, we apply the following algorithm:

$O^{n+1}=O^{n}+\mathcal{T}^{-1}\left(W-\left(P_{\mathrm{w}} \circ \mathcal{T}\right) P * O^{n}\right)$

where $\mathcal{T}^{-1}$ is the inverse wavelet transform.

1. Set $n$ to 0 .
2. Find the initial estimation $O^{n}$ by applying an inverse wavelet transform to the set $W$ corresponding to the detected wavelet coefficients in the data.

3. Convolve $O^{n}$ with the PSF $P: I^{n}=P * O^{n}$.

4. Determine the wavelet transform $W\left(I^{n}\right)$ of $I^{n}$.

5. Threshold all wavelet coefficients in $W\left(I^{n}\right)$ at position and scales where nothing has been detected (i.e. $P_{\mathrm{w}}$ operator). We get $W_{\mathrm{t}}\left(I^{n}\right)$.

6. Determine the residual $W(R)=W-W_{\mathrm{t}}\left(I^{n}\right)$.

7. Reconstruct the residual image $R^{n}$ by applying an inverse wavelet transform.

8. Add the residual to the solution: $O^{n+1}=O^{n}+R^{n}$.

9. Threshold negative values in $O^{n+1}$.

10. If $\sigma\left(R^{n}\right) / \sigma\left(O^{0}\right)<\epsilon$ then $n=n+1$ and go to step 3 . 11. $O^{n+1}$ contains the deconvolved reconstructed object.

In practice, convergence is very fast (less than 20 iterations). The reconstructed image (not deconvolved) can also be obtained just by reconvolving the solution with the PSF.

\subsection{Space-variant PSF}

Deconvolution methods generally do not take into account the case of space-variant PSF. The standard approach when the PSF varies is to decompose the image into blocks, and to consider the PSF constant inside a given block. Blocks which are too small lead to a problem of computation time (the FFT cannot be used), while blocks which are too large introduce errors due to the use of an incorrect PSF. Blocking artifacts may also appear. Combining source detection and deconvolution opens up an elegant way for deconvolution with a space-variant PSF. Indeed, a straightforward method is derived by just replacing the constant PSF at step 3 of the algorithm with the PSF at the centre of the object. This means that it is not the image which is deconvolved, but its constituent objects.

\subsection{Deconvolution and resolution}

\subsubsection{The intrinsic correlation function}

In many cases, there is no sense in trying to deconvolve an image at the resolution of the pixel (especially when the PSF is very large). The idea to limit the resolution is relatively old, because it is already this concept which is used in the CLEAN algorithm (Högbom 1974). Indeed the Clean-Beam fixes the resolution in the final solution. This principle was also developed by Lannes (1987) in a different form. This concept has been re-invented, first by Gull \& Skilling (1991) who have called the Clean-Beam the Intrinsic Correlation Function (ICF), and more recently by Magain (1998) and Pijpers (1999).

The ICF is usually a Gaussian, but in some cases it may be useful to take another function. For example, if we 
want to compare two images $I_{1}$ and $I_{2}$ which are obtained with two wavelengths or with two different instruments, their PSFs $P_{1}$ and $P_{2}$ will certainly be different. The classic approach would be to deconvolve $I_{1}$ with $P_{2}$ and $I_{2}$ with $P_{1}$, so we are sure that both are at the same resolution. But unfortunately we lose some resolution in doing this. Deconvolving both images is generally not possible because we can never be sure that both solutions $O_{1}$ and $\mathrm{O}_{2}$ will have the same resolution.

A solution would be to deconvolve only the image which has the worse resolution (say $I_{1}$ ), and to limit the deconvolution to the second image resolution $\left(I_{2}\right)$. Then, we just have to take $P_{2}$ for the ICF. The deconvolution problem is to find $\tilde{O}$ (hidden solution) such that:

$I_{1}=P_{1} * P_{2} * \tilde{O}$

and our real solution $O_{1}$ at the same resolution as $I_{2}$ is obtained by convolving $\tilde{O}$ with $P_{2} . O_{1}$ and $I_{2}$ can then be compared.

Introducing an ICF $G$ in the deconvolution equation leads to just considering a new PSF $P^{\prime}$ which is the convolution of $P$ and $G$. The deconvolution is carried out using $P^{\prime}$, and the solution must be reconvolved with $G$ at the end. In this way, the solution has a constrained resolution, but aliasing may occur during the iterative process, and it is not sure that the artifacts will disappear afer the re-convolution with $G$. Magain (1998) has proposed an original alternative to this problem, by assuming that the PSF can be considered as the convolution product of two terms, the ICF $G$ and an unknown $S, P=G * S$. Using $S$ instead of $P$ in the deconvolution process, and a sufficiently large $F W H M$ value for $G$, implies that the Shannon sampling theorem (Shannon 1948 ) is never violated. But the problem is now to calculate $S$, knowing $P$ and $G$, which is again a deconvolution problem. Unfortunately, this delicate point was not discussed in the original paper. Propagation of the error on the $S$ estimation in the final solution has also until now not been investigated, even if this issue seems to be quite important.

\subsubsection{ICF calculation}

This section describes how to calculate the $F W H M$ for a given sampling, in order not to violate the Shannon sampling theorem. Gaussian functions are generally chosen for the ICF. The resolution to be achieved is fixed by its standard deviation $\sigma_{\mathrm{G}}$, or its $F W H M\left(F W H M=2.34 \sigma_{\mathrm{G}}\right)$. Since the Fourier transform of a Gaussian of standard deviation $\sigma_{\mathrm{G}}$ is also a Gaussian of standard deviation $\sigma_{\nu}=\frac{N}{2 \pi \sigma_{\mathrm{G}}},(N$ being the number of pixels $)$, we can estimate the smallest $F W H M$ which does not violate the Shannon sampling theorem. In theory, a Gaussian cannot respect it, but in practice we can consider that values smaller than a given $\epsilon$ have no practical effect, and the
Table 1. ICF standard deviation

\begin{tabular}{ccc}
\hline \hline$\epsilon$ & ICF $\sigma_{\mathrm{G}}$ & ICF $F W H M$ \\
\hline \hline $10^{-3}$ & 1.18 & 2.77 \\
$10^{-4}$ & 1.37 & 3.20 \\
$10^{-5}$ & 1.53 & 3.57 \\
$10^{-7}$ & 1.81 & 4.23 \\
$10^{-10}$ & 2.16 & 5.05 \\
$10^{-20}$ & 3.05 & 7.15 \\
\hline \hline
\end{tabular}

Shannon sampling theorem is experimentally respected if $\exp -\frac{u^{2}}{2 \sigma_{\nu}^{2}}<\epsilon$ when $u>\frac{N}{2}$.

For $u=\frac{N}{2}$, we have: $\exp -\frac{\pi^{2} \sigma_{\mathrm{G}}^{2}}{2}<\epsilon$.

Then the smallest ICF standard deviation $\sigma_{\mathrm{G}}$ is given by

$\sigma_{\mathrm{G}}=\sqrt{-\frac{2 \log \epsilon}{\pi^{2}}}$

Table 1 gives the $\sigma_{\mathrm{G}}$ values for different values of $\epsilon$. If the resolution to be achieved is smaller than $\sigma_{\mathrm{G}}$, this means that the solution sampling must be fainter than the data sampling.

\subsubsection{Undersampled point spread function}

If the PSF is undersampled, it can be used in the same way, but results may not be optimal due to the fact that the sampled PSF varies depending on the position of the source. If an oversampled PSF is available, resulting from theoretical calculation or from a set of observations, it should be used to improve the solution. In this case, each reconstructed object will be oversampled. Equation (4) must be replaced by

$J(O)=\left\|W-\left(P_{\mathrm{w}} \circ \mathcal{T} \circ \mathcal{D}_{l}\right) P * O\right\|$

where $\mathcal{D}_{l}$ is the averaging-decimation operator, consisting of averaging the data in the window of size $l \times l$, and keeping only one average pixel for each $l \times l$ block.

\section{Application to ISOCAM data}

\subsection{ISOCAM point source reconstruction}

A simulation was performed in order to analyse how well the flux is estimated. A point source (using ISOCAM 6 arcsec lens PSF) was simulated, with a constant background (value of 100), and uniform Gaussian noise $($ sigma $=1)$. The integrated flux of the sources varies from 7 to 102 . Table 2 gives the results of this simulation. The first column gives the real flux, the second column the flux found using MVM, and the third column the flux found using MVM plus the deconvolution (MVMD). The photometry is clearly improved using MVMD. Another aspect of MVMD is that the error is relatively constant, whatever the flux of the source. 

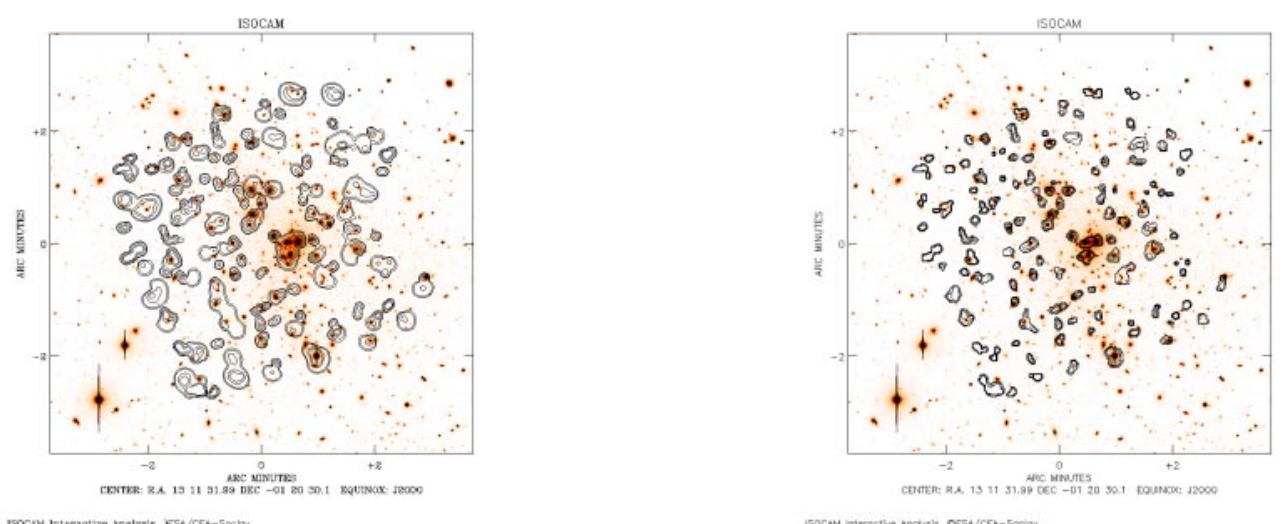

Fig. 3. Abell 1689: left, ISOCAM source detection (isophotes) overplotted on an optical image (NTT, band $V$ ). The ISOCAM image is a raster observation at $7 \mu \mathrm{m}$. Right, ISOCAM source detection using the PSF (isophotes) overplotted on the optical image. Compared to the left panel, it is clearly easier to identify the detected infrared sources in the optical image
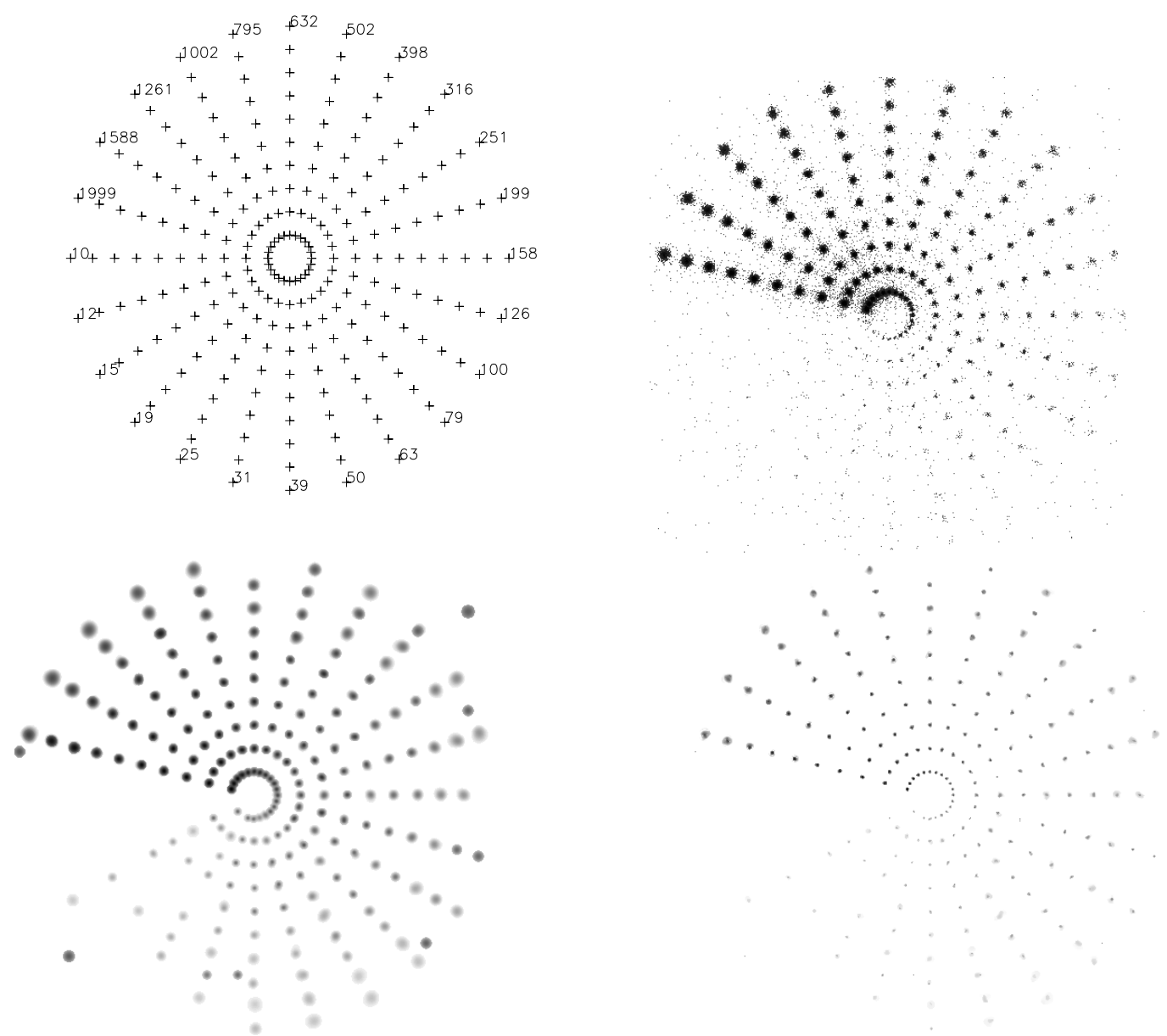

Fig. 4. XMM model simulation. Upper left, position of the point sources. The flux is the same on each radial line. Upper right, simulated data with Poisson noise. The background is $\sim 0.1$ counts/pixel. Bottom left and right shows respectively the result of the detection by the MVM with and without the PSF model 
Table 2. Flux estimation from MVM and MVM + deconvolution (MVMD)

\begin{tabular}{ccccccc}
\hline \hline Real Flux & MVM Flux & MVMD Flux & MVM Error & MVM Error (\%) & MVMD Error & MVMD Error (\%) \\
\hline 7 & 7.35 & 5.96 & 5.00 & 14.82 & 0.35 & 1.03 \\
12 & 12.66 & 12.62 & 5.51 & 5.21 & 0.66 & 0.62 \\
17 & 17.57 & 18.41 & 3.37 & 8.30 & 0.57 & 1.41 \\
22 & 21.68 & 23.42 & 1.41 & 6.46 & 0.31 & 1.42 \\
27 & 26.18 & 28.43 & 3.00 & 5.31 & 0.81 & 1.43 \\
32 & 30.74 & 33.44 & 3.93 & 4.51 & 1.25 & 1.44 \\
37 & 35.28 & 38.45 & 4.64 & 3.93 & 2.08 & 1.45 \\
42 & 39.91 & 43.46 & 4.95 & 3.49 & 2.39 & 1.47 \\
47 & 44.60 & 48.47 & 5.09 & 3.13 & 2.75 & 1.48 \\
52 & 49.24 & 53.48 & 5.29 & 2.85 & 3.06 & 1.49 \\
57 & 53.93 & 58.49 & 5.37 & 2.61 & 3.32 & 1.49 \\
62 & 58.67 & 63.49 & 5.36 & 2.41 & 3.85 & 1.50 \\
67 & 63.42 & 68.50 & 5.33 & 2.24 & 4.06 & 1.51 \\
72 & 68.14 & 73.51 & 5.35 & 2.09 & 4.29 & 1.52 \\
77 & 72.93 & 78.51 & 5.27 & 1.96 & 4.52 & 1.52 \\
82 & 77.70 & 83.52 & 5.23 & 1.85 & 4.75 & 1.53 \\
87 & 82.47 & 88.52 & 5.20 & 1.75 & 4.97 & 1.53 \\
92 & 87.24 & 93.53 & 5.16 & 1.66 & 5.15 & 1.53 \\
97 & 92.02 & 98.53 & 5.13 & 1.58 & & \\
102 & 96.84 & 103.5 & 5.05 & 1.50 & & \\
\hline \hline
\end{tabular}

\subsection{Abell 1689 ISOCAM data}

Figure 3 (left) shows the detections (isophotes) obtained using the MVM method without deconvolution on ISOCAM data. The data were collected using the 6 arcsec lens at $6.75 \mu \mathrm{m}$. This was a raster observation with $10 \mathrm{~s}$ integration time, 16 raster positions, and 25 frames per raster position. The noise is non-stationary, and the detection of the significant wavelet coefficients was carried out using the root mean square error map $R_{\sigma}(x, y)$ by the method described in Starck et al. (1999). The isophotes are overplotted on an optical image (NTT, band $V$ ) in order to identify the infrared source. Figure 3 (right) shows the same treatment but using the MVM method with deconvolution. The objects are the same, but the photometry is improved, and it is clearly easier to identify the optical counterpart of the infrared sources.

\section{Application to XMM simulations}

\subsection{Introduction}

One case in astronomical imagery where the PSF varies across the field of view (FOV) is with space X-ray telescope observations. In order to illustrate our methods we have selected one example from an ESA space mission, the X-ray Multi-Mirror Telescope (XMM). One feature of the X-ray images is that they are in a photon noise regime - practically the incoming photons on the detector are counted one by one and their energy is recorded. The resulting photon event list can be binned into an image by choosing both the pixel size and the energy band.
The response of the telescope for incoming light from a point source (the PSF) depends on the position of the source across the FOV and also depends on the energy passband. What makes the task of object detection and reconstruction of the parameters difficult can be summarized as follows:

- few photons spread over a large area;

- object classification problems (AGN/point-like or extended) due to the non-constant PSF, and

- source confusion - large extended objects overlayed with many point-like sources.

\subsection{Poisson noise modelling}

If a wavelet coefficient $w_{j}(x, y)$ is due to noise, it can be considered as a realization of the sum $\sum_{k \in K} n_{k}$ of independent random variables with the same distribution as that of the wavelet function $\left(n_{k}\right.$ being the number of events used for the calculation of $\left.w_{j}(x, y)\right)$. This allows comparison of the wavelet coefficients of the data with the values which can be taken by the sum of $n$ independent variables.

The distribution of one event in wavelet space is then directly given by the histogram $H_{1}$ of the wavelet $\psi$. Since we consider independent events, the distribution of a coefficient $w_{n}$ (note the changed subscripting for $w$, for convenience) related to $n$ events is given by $n$ autoconvolutions of $H_{1}$ :

$H_{n}=H_{1} \otimes H_{1} \otimes \ldots \otimes H_{1}$.

For a large number of events, $H_{n}$ converges to a Gaussian. More details can be found in Starck \& Pierre (1998). 

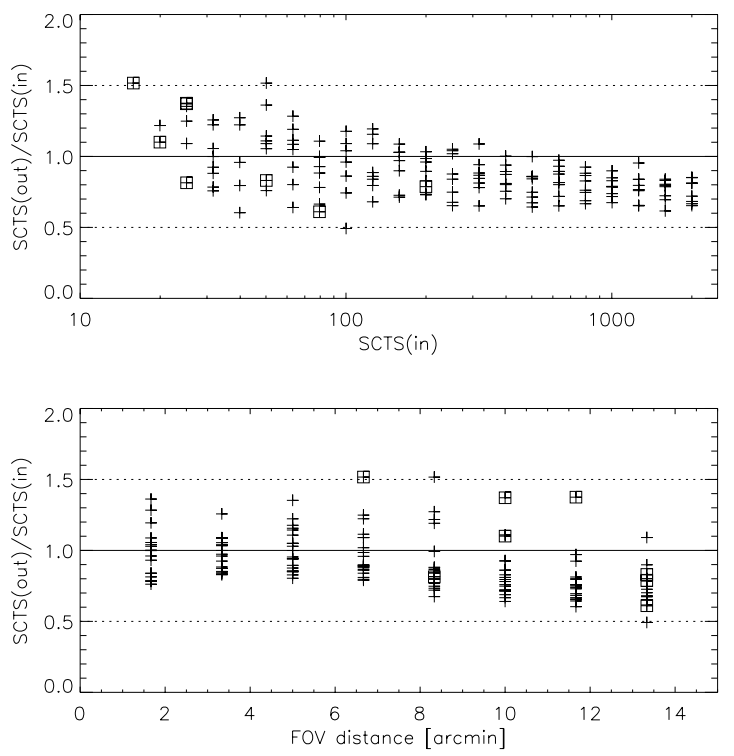

Fig. 5. XMM model simulation. Results for the detections. The cross-identification searching radius was set to $12^{\prime \prime}$ and the limiting distance from the centre to $14^{\prime}$. The ratio of the detected counts $(S C T S$ (out)) to the input source counts (SCTS(in)) is shown as a function of $S C T S$ (in) (upper panel) and as a function of the off-axis distance (lower panel). The ratios of $1,0.5$ and 1.5 are indicated with continuous and dashed lines. Also the objects with distance greater than $4^{\prime \prime}$ from their corresponding object are indicated with squares

\subsection{Results}

In our XMM example, we created simulated images including most of the telescope effects - PSF blurring, vignetting effect, particle and instrumental background. Our objective was to test the ability of the method to deconvolve images with a space-variant PSF. The image contains a set of point sources at different positions and with different fluxes. An energy band from 0.4 to $4.0 \mathrm{keV}$ was used but this is irrelevant for our main aim. Figure 4, upper left, shows the position of the sources. On each radial line, the flux in the sources is identical, but the PSF becomes larger when the distance from the centre increases and the number of lost photons due to vignetting could well reach 50\%. Fluxes run from 10 at left and counterclockwise with logarithmic step to $2000([10,12,15,19, \ldots, 1002,1261,1588,1999])$. Figure 4, upper right, shows the simulated data. The background is $10^{-5}$ counts/pixel/second, so for $10 \mathrm{ks}$ this corresponds to $\sim 0.1$ counts/pixel. Figure 4 , bottom left and right, show respectively the result of the detection by the MVM with and without the PSF. Figure 5 shows the recovery of fluxes for the input sources after correction for the vignetting effect.

One example of a "realistic" image, with extended as well as point-like sources is shown in Fig. 6 and the

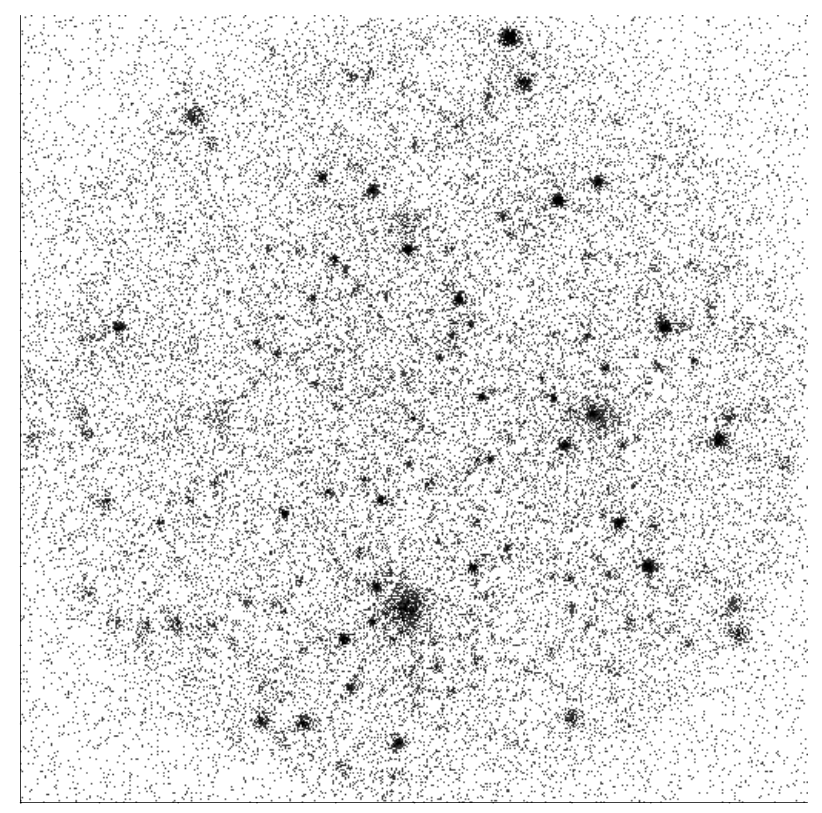

Fig. 6. Realistic X-ray image. The exposure time is $10000 \mathrm{~s}$, the point-like sources are distributed according to a $\log N-\log S$ relation (Hasinger et al. 1998), there are 5 extended sources clusters of galaxies at 5 different redshifts $(0.6,1,1.5,1.8$ and 2 ) with King $\beta$-model profiles and temperature $5 \mathrm{keV}$

reconstruction after deconvolution with MVM and PSF in Fig. 7.

The recovery of the input $\log N-\log S$ relation for the point-like sources is shown in Fig. 8 together with the distribution and the numbers of missing input objects and false detections.

Note that in order to perform the cross-identifications with the input list we take all the input sources with photon counts greater than 13 . That is a rather low limit and it is the reason for the large number of missed detections.

More comprehensive analysis and comparison of this method with other methods dedicated to detection of objects in XMM-specific X-ray images can be found in Valtchanov (2000).

\section{Application to Chandra simulations}

Our experiments on Chandra simulations allowed us to look at the computational requirements of our method. The detect toolkit (Dobrizycki et al. 1999) was designed for analysis of Chandra X-ray Observatory data. We used a simulation (see Fig. 9) from the HRC-I High Resolution Camera imaging detector. The field of view of HRC-I is 31 arcmin $\times 31$ arcmin. The background corresponds to a $30 \mathrm{ks}$ exposure. The simulation shows 6 repetitions of an aligned set of unresolved sources of the same intensity. From the first source, the following 5 are spaced at 0.5 , 4, 5, 10 and 30 arcsec. From the last of these, at 60 arcsec distance, the same aligned pattern is repeated, but a large extended source is superimposed. The extended 


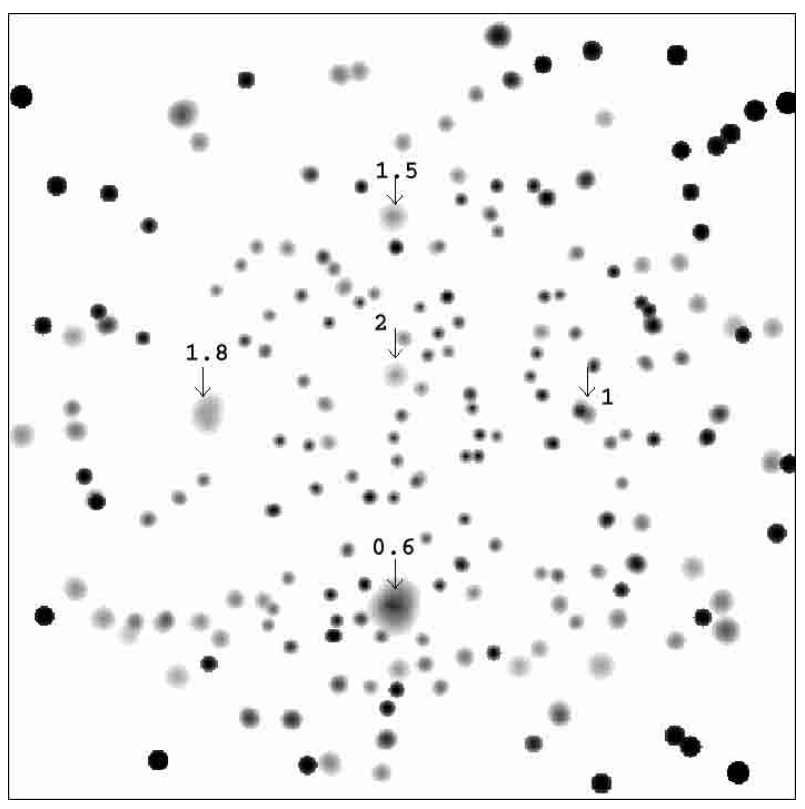

Fig. 7. Realistic X-ray image. The detected objects with $4 \sigma$ significance are shown and the extended objects are indicated by an arrow and the redshift

source is a Gaussian with sigma 25 arcsec. In addition to the aligned set of sources just described, they are also displayed shifted to the right, in parallel, by 60 arcsec and 120 arcsec, providing three parallel rows. The first row is much clearer: the sources have approximately 200 counts each. The extended source has about 2500 counts. In the second row, the sources are much weaker, having about 30 counts each. The third row is the same as the middle row, except that the sources are changed to disks with diameter 1 arcsec.

Detection of sources which are within 1 arcsec of each other was not achieved by the detection methods described in Dobrzycki et al. (1999) and will not be investigated here either. This implies that of the six sources considered in each half-row of the simulation, in effect four were used. Figure 9 appears, therefore, to have a total of 24 compact sources. Likewise the extended sources were not considered in Dobrzycki et al. (1999), nor by us.

In Dobrzycki et al. (1999), two methods for source detection based on wavelet transforms are described. The first, the celldetect method, is based on a sliding cell or window. Its performance is good, finding 22 of the 24 nonextended sources in the simulation. It is fast but requires a considerable range of parameters to be set by the user. A multiple scale binning procedure is used to handle very large images. It allows for variation in bin size to cater for off-axis detector spatial variation.

We selected an isolated point source, to which we fitted a Gaussian, to define a very approximate PSF. We used an 8 resolution scale $B_{3}$ spline à trous wavelet transform, with a Poisson noise model. We worked directly on the $2800 \times 2800$ array, with no preprocessing. In view of the

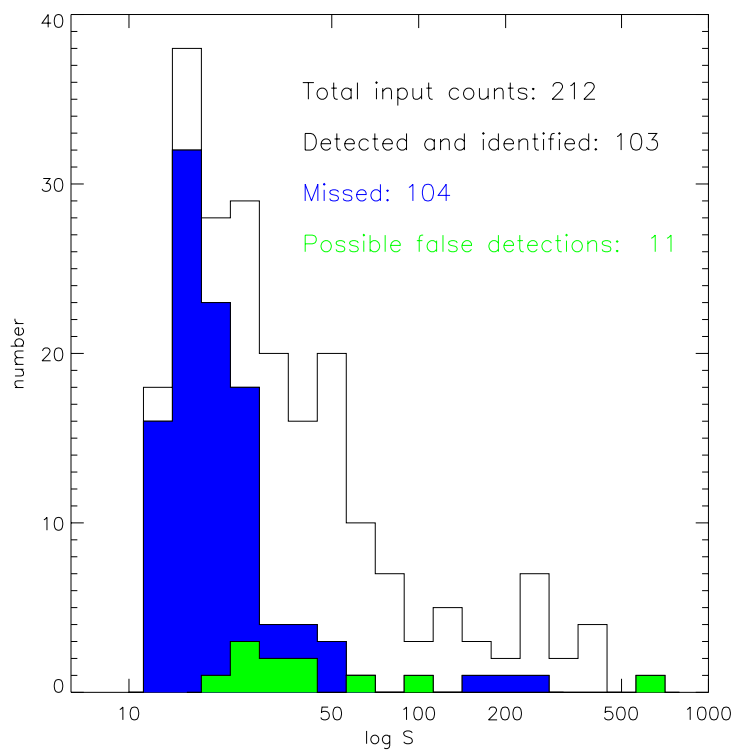

Fig. 8. Realistic X-ray image. The input $\log N-\log S$ relation together with the distribution of the non-detected input sources (blue histogram) and the distribution of the possible false detections (in green). Note however that the extended sources here are counted as false detections

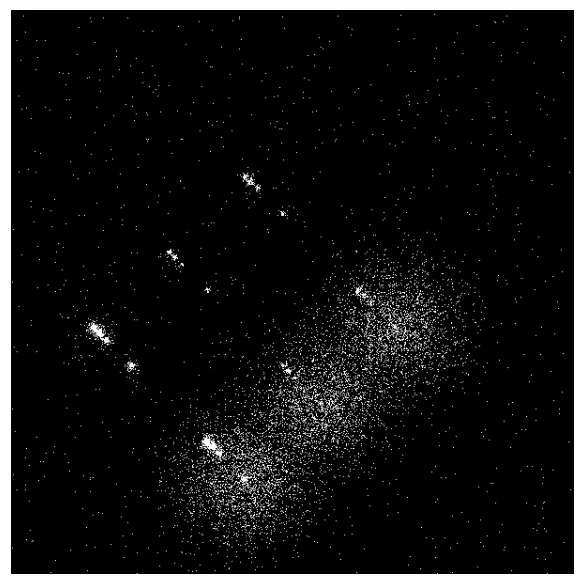

Fig. 9. Simulated HRC-I image, original of dimensions $2800 \times$ 2800

faintness of some of the sources which we are trying to detect, we set a very low threshold, 1.5 sigma.

Figure 10 shows ellipses fitted to sources detected. Positions of detections are shown in Fig. 11, and an associated table of parameters contains information on the objects detected. The approach used here, which is based on resolution scale and noise modelling, and in addition carries out deconvolution, requires very little by way of user setting of parameters.

The analysis of Chandra data is made difficult by offaxis variation in the PSF, which we have not taken into account. Indeed, the PSF becomes bimodal when far offaxis. The wavdetect procedure provided in the detect image analysis software package is based on a Mexican 


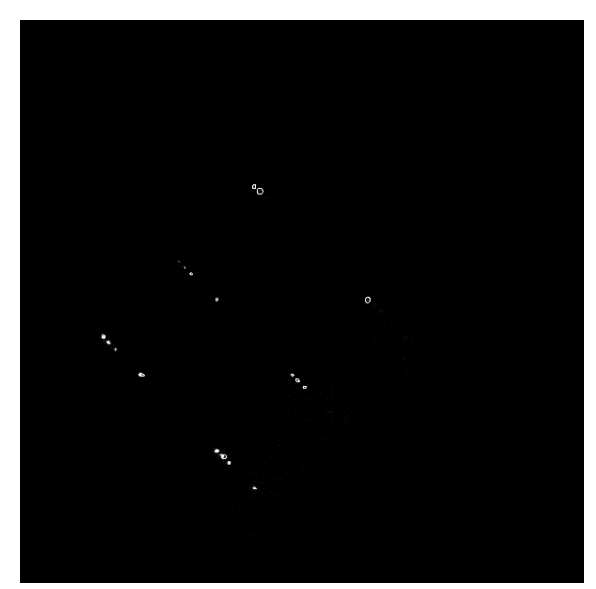

Fig. 10. Source detections, marked

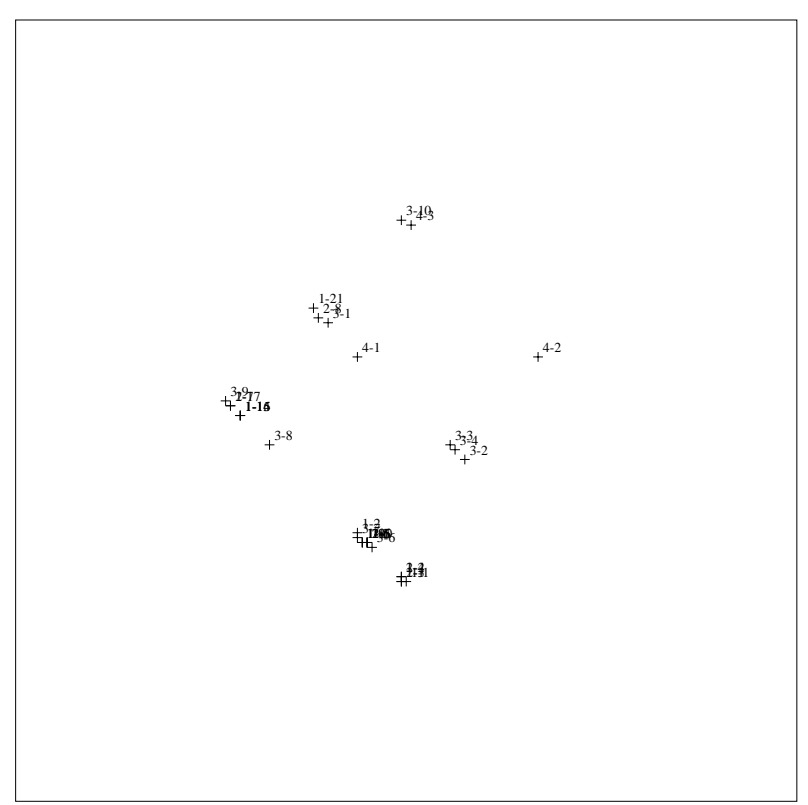

Fig. 11. Source detections, labelled

hat wavelet function. Its storage requirements result in an image size of $1024 \times 1024$ being recommended as the practical limit to be used. Our own code incorporates memory management and we analyzed, without prior blocking, a $2800 \times 2800$ image.

Examination of a smaller field shown in Fig. 12 allows us to go further, - to distinguish between sources which are within 1 arcsec. Figures 13 and 14 show the objects found and a sub-object analysis.

\section{Conclusion}

We have shown that the source detection can be combined with a deconvolution when using wavelet based methods such as the Multiscale Vision Model. This leads to the reconstruction of deconvolved objects. The main advantages

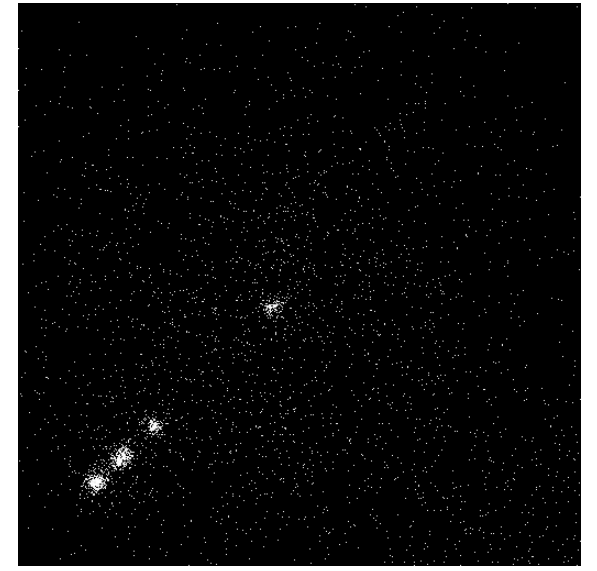

Fig. 12. Part of the larger Chandra simulation

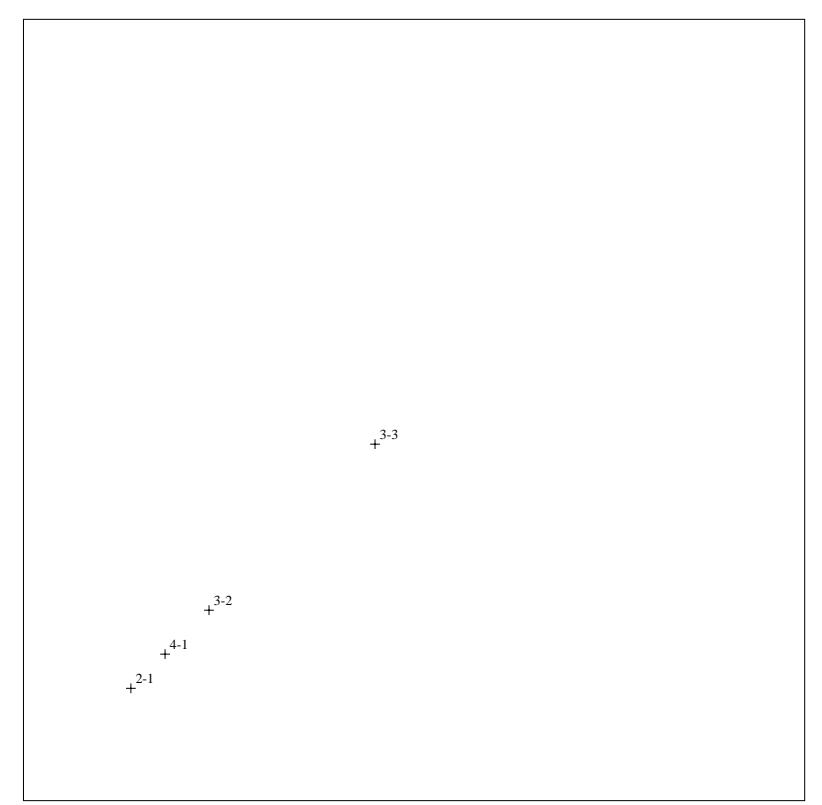

Fig. 13. Locations of detected objects

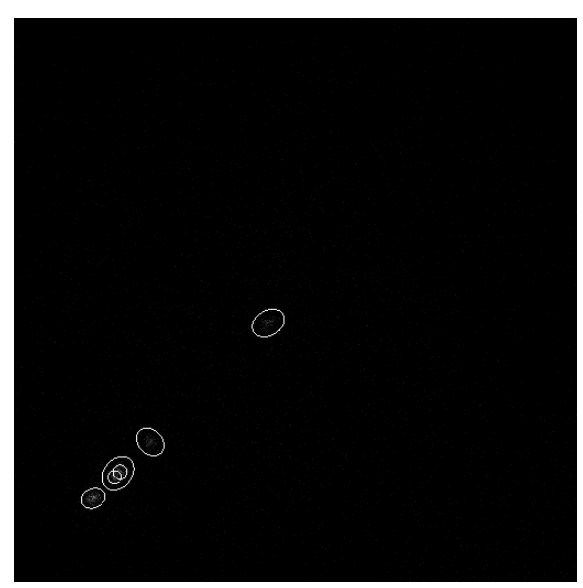

Fig. 14. Locations of detected objects, following sub-object analysis, with overplotted ellipses 
of this approach are:

- Faint extended objects can be detected as well as point sources;

- The analysis does not require background estimation. (We know that if the background varies spatially, its estimation becomes a non-trivial task and may produce large errors in object photometry);

- Objects are easier to identify in the deconvolved map;

- Morphological parameters (gallaxy ellipticity and so on) are more accurate;

- Since each object is deconvolved separately, a spatially variable point spread function can easily be taken into account;

- Very large images can be deconvolved.

We may note that observing, in practice, requires consideration of issues prior to, or beyond, what has been considered in this work. Examples include: extensive detector imperfections, the managing of multiple wavelength observations, observations requiring knowledge of physical phenomena. All of these produce observational errors, which we have not considered. Our aim has instead been to futher deconvolution and detection technologies. Towards this end, we have considered a wide range of practical observing cases, but certainly not all.

The software used in this paper is based on that described in Starck et al. (1998) and is implemented in the MR/1 multiresolution analysis environment available through the website http://www.multiresolution.com

Acknowledgements. We would like to thank Dan Harris, CfA, for the simulated Chandra data. This work was partially supported by U.S.A. National Science Foundation grant DMS 98-72890 (KDI).

\section{References}

Bijaoui A., 1993, in Meyer Y. and Roques S. (eds.), Progress in wavelet analysis and applications. Éditions Frontières, pp. $551-556$

Bijaoui A., Rué F., 1995, Signal Proc. 46, 229

Dobrzycki A., Ebeling H., Glotfelty K., Freeman P., Damiani F., Elvis M., Calderwood T., 1999, CHANDRA detect 1.1 User Guide, Technical report, Chandra X-Ray Center, Smithsonian Astrophysical

Gonzalez R.C., 1993, Digital Image Processing. AddisonWesley

Gull S., Skilling J., 1991, MEMSYS5 Quantified Maximum Entropy User's Manual

Hasinger G., Burg R., Giacconi R., et al., 1998, A\&A, 329, 482

Högbom J., 1974, A\&AS 15, 417

Lannes A., Roques S., 1987, J.O.S.A. 4, 189

Magain P., Courbin F., Sohy S., 1998, AJ 494, 472

Narayan R., Nityananda R., 1986, ARA\&A 24, 127

Pijpers F.P., 1999, MNRAS 307, 659

Pratt W., 1991, Digital Image Processing. Wiley

Rué F., Bijaoui A., 1997, Exp. Astron. 7, 129

Shannon C., 1948, Bell Syst. Tech. J. 27, 379

Starck J., Aussel H., Elbaz D., Fadda D., Cesarsky C., 1999, A\&AS 138, 365

Starck J., Murtagh F., Bijaoui A., 1998, Image Processing and Data Analysis: The Multiscale Approach. Cambridge University Press, Cambridge (UK)

Starck J., Pierre M., 1998, A\&AS 128, 397

Valtchanov I., 2000, A\&A (in preparation) 